Editorial

\title{
Radiation therapy's role in multidisciplinary and patient centric care
}

Volume I Issue 2 - 2014

As a senior radiation therapist at Wellspan Health's York Cancer Center in York, $\mathrm{Pa}$, I have the great privilege to work with an amazing group of therapists whose main focus is that of a Patient Centric approach in their role as a Multidisciplinary team member. I have had the distinct pleasure of working with outstanding Radiation Therapist's who take on the role of patient advocate with great vigor and caring. Our main goal is to get the patient through this time in their lives, this very personal and often private time that most never thought would happen to them. This is amazing and humbling presence, as a radiation therapist, plays a great role in the Multidisciplinary process of cancer care.

From the first day the patient comes into our offices for their CT simulation, a radiation therapist is there to ease their way into the treatment process, answering questions from the patient and family on what to expect during today's simulation as well as their daily treatments. On the first day of simulation so many of our patients are apprehensive and filled with anxiety, I believe that it is our great honor to get them through this first "Giant" step in their cancer treatment process. Many of our patients become attached to their "Simulation" therapist as they were the first staff member to guide them through their amazingly personal experience, that is, their radiation therapy cancer treatments. As therapists we are the daily connection to the patient and are at the forefront of Patient Centric Care. We are their advocates on a daily basis to voice their issues and concerns in between OTV appointments.

Once a patient has started their daily treatments we as therapists play an intricate role in the Multidisciplinary and Patient Centric Care process. Seeing our patients five days a week we have a great insight into how they are feeling on a daily basis, we can see the small changes in their behavior as well as their general well being and can get the intervention they may need quickly to alleviate their pain, anxiety, dehydration and psycho-social issues that may delay or interfere with treatment. With so many of our patients receiving chemotherapy as well as radiation therapy we have a great deal of intra-departmental collaboration to ensure our patients are receiving their therapies as needed. Dealing with multiple sites that deliver the chemotherapy to our patients there needs to be great communication and collaboration with the various sites to allow for the different chemotherapy regimens and the often long hours that the patients are in the departments for their infusions. This week in the North East is a great example, with all of the snow that has blanketed our area, some patient's chemotherapy treatments were canceled and some patients were unable to travel to our center due to the weather. Without the excellent lines of communication we have in place and

\author{
Bryan M Schmalhofer \\ Wellspan Health, USA
}

Correspondence: Bryan M Schmalhofer,Wellspan Health, 323

S. West End Ave, Lancaster, Pa 17603 Work: 25 Monument Rd.

Suite 94, York, Pa 17403, USA, Tel 717-3 I 4-2227,

Email bschmalh@eastern.edu

Received: August 20, 2014 | Published: August 25, 2014

the roles that our therapists play in ensuring that our patients receive both their chemotherapy and radiation therapy as prescribed, the multidisciplinary care model would fail under these circumstances.

Being on the front line in the patient centric care environment we have the distinct advantage of getting to know our patients and can often times intervene when they need the services of our social workers, nurses, financial counselors, clergy and transportation coordinators. An additional role we play in the Multidisciplinary and Patient Centric Care of our patients is with the communication with our dietary staff. With the majority of our patients receiving CBCT daily prior to treatment we are able to tell at a very early stage if our patients are losing weight by looking at the patient's contours on the $\mathrm{CBCT}$ as compared to the initial planning CT. This early intervention can lead to closer following of the patient by the dietary staff to head off any rapid weight loss. We can also see the changes on a daily basis in tumor reduction that is causing patients anatomy to shift and not line up correctly and often precipitates the need for a re-planning CT simulation to correct for the change in tumor volume.

Having had the great privilege of working with our patients at their greatest emotional and physical needs, we as therapists take great pride in our role as a member of the Multidisciplinary and Patient Centric care team. In our ever changing healthcare environment Multidisciplinary and Patient Centric care will continue to be the model of the future for our patients and the therapist's role will be ever more intricate and involved.

\section{Acknowledgments}

None.

\section{Conflicts of interest}

Author declares there are no conflicts of interest. 